\title{
FINITE ELEMENT ANALYSIS OF MICROSTRUCTURE FOR THE CUBIC TO TETRAGONAL TRANSFORMATION*
}

\author{
$\mathrm{BO} \mathrm{LI}^{\dagger}$ AND MITCHELL LUSKIN $\ddagger$
}

\begin{abstract}
Martensitic crystals which can undergo a cubic to tetragonal phase transformation have a nonconvex energy density with three symmetry-related, rotationally invariant energy wells. We give estimates for the numerical approximation of a first-order laminate for such martensitic crystals. We give bounds for the $L^{2}$ convergence of directional derivatives in the "twin" plane, for the $L^{2}$ convergence of the deformation, for the weak convergence of the deformation gradient, for the convergence of the microstructure, and for the convergence of nonlinear integrals of the deformation gradient.
\end{abstract}

Key words. finite element, nonconvex variational problem, microstructure, martensitic transformation, error estimate

AMS subject classifications. 49M15, 65C20, 65N30, 73C50, 73G05, 73K20

PII. S0036142996301111

1. Introduction. Martensitic crystals have a high temperature, symmetric solid phase known as austenite, and a lower temperature, less symmetric solid phase known as martensite. The austenitic phase exists in one variant, but the martensitic phase can exist in several symmetry-related variants. For some boundary conditions, the elastic energy of a martensitic crystal can be lowered as much as possible only by the fine scale mixing of the martensitic variants to form a microstructure. A simple and common example of such a microstructure is a first-order laminate in which the deformation gradient oscillates in parallel layers between two stress-free homogeneous states.

Based on the assumption that the crystal structure is determined by the principle of energy minimization, the recently developed geometrically nonlinear theory of thermoelasticity describes the martensitic microstructure as the limit of energyminimizing sequences of deformations; see $[2,3,13,16,19,20]$ and the references therein. In this theory, the elastic energy density for the crystal below the transformation temperature is nonconvex and is minimized on a set of deformation gradients $\mathrm{SO}(3) U_{1} \cup \cdots \cup \mathrm{SO}(3) U_{M}$ for $M>1$, where $\mathrm{SO}(3)$ is the set of proper rotations, that is,

$$
\operatorname{SO}(3)=\left\{Q \in \mathbb{R}^{3 \times 3}: Q^{T}=Q^{-1} \text { and } \operatorname{det} Q=1\right\}
$$

where $\mathbb{R}^{3 \times 3}$ is the set of all $3 \times 3$ real matrices, and where the $U_{i} \in \mathbb{R}^{3 \times 3}$ represent the symmetry-related martensitic variants. Martensitic crystals that can undergo a cubic to tetragonal phase transformation have three martensitic energy wells, so $M=3$ $[2,3,20]$.

* Received by the editors March 25, 1996; accepted for publication (in revised form) October 8, 1996. This work was supported in part by the NSF through grants DMS 91-11572 and DMS 9505077, by the ARO through grant DAAL03-92-G-0003, by the Institute for Mathematics and Its Applications, and by a grant from the Minnesota Supercomputer Institute.

http://www.siam.org/journals/sinum/35-1/30111.html

$\dagger$ Department of Mathematics, University of California at Los Angeles, Los Angeles, CA 900951555 (bli@math.ucla.edu).

${ }^{\ddagger}$ School of Mathematics, University of Minnesota, 206 Church Street, S.E., Minneapolis, MN 55455 (luskin@math.umn.edu). 
We present in this paper a numerical analysis of the approximation of an energyminimizing first-order laminate for the cubic to tetragonal transformation. Ball and James have shown for boundary conditions that are consistent with such a first-order laminate that no energy-minimizing microstructure other than the first-order laminate can exist [3]. We establish in this paper an approximation theory for the cubic to tetragonal transformation with bounds for the $L^{2}$ convergence of directional derivatives in the "twin plane," for the $L^{2}$ convergence of the deformation, for the weak convergence of the deformation gradient, for the convergence of the microstructure, and for the convergence of nonlinear integrals of the deformation gradient. These bounds are then used to give error estimates for conforming finite element approximations. Three-dimensional numerical computations for the cubic to tetragonal phase transformation have been reported in $[11,17,18]$, and the numerical analysis presented in this paper gives a rigorous validation for some of the reported numerical experiments.

A theory of numerical analysis for the microstructure in nonconvex variational problems was developed in $[10,12]$ and has been extended in $[4,6,7,8,15,24]$. Analyses of the approximation of relaxed variational problems have been given in $[5,14,25,26,27,29,30]$. Our work relies most directly on the analysis given in [21] for the numerical approximation of microstructure for a rotationally invariant, double well energy density. We note that the analysis given in [21] covers the orthorhombic to monoclinic transformation which can be modeled by a double well energy density $[3,20]$. An extension of the analysis in this paper to a class of nonconforming elements is given in [22].

We refer to the recent paper [20] for a more extensive survey on the numerical computation and analysis of martensitic microstructure. We also refer to [23] for an analysis of the convergence of numerical methods for the computation of microstructure in ferromagnetic crystals [23].

In section 2 we first describe the underlying continuum model for the cubic to tetragonal martensitic transformation. We prove in section 3 the convergence in $L^{2}$ for the directional derivatives in the "twin plane" of energy-minimizing sequences of deformations, and we prove in section 4 the convergence in $L^{2}$ of the deformation and the weak convergence of the deformation gradient. In section 5 we prove the convergence of the microstructure for energy-minimizing sequences of deformations. More precisely, we give bounds for the volume fractions of the oscillating deformation gradients, and we prove the convergence of nonlinear integrals of the deformation gradient. Finally, in section 6 we present error estimates for conforming finite element approximations.

2. The continuum model. We denote by $\Omega$ the reference configuration of the crystal, which is taken to be the homogeneous austenitic state at the transformation temperature. We assume that $\Omega \subset \mathbb{R}^{3}$ is a bounded domain with a Lipschitz continuous boundary. We denote deformations by $y: \Omega \rightarrow \mathbb{R}^{3}$ and corresponding deformation gradients by $\nabla y: \Omega \rightarrow \mathbb{R}^{3 \times 3}$. We denote the elastic energy density at a fixed temperature below the transformation temperature by the continuous function $\phi: \mathbb{R}^{3 \times 3} \rightarrow \mathbb{R}$. We consider the variational problem to minimize the elastic energy

$$
\mathcal{E}(y) \equiv \int_{\Omega} \phi(\nabla y(x)) d x
$$

over all deformations $y$ which satisfy given boundary conditions. 
The variants of the tetragonal phase for the cubic to tetragonal transformation can be given by $[2,3,20]$

$$
\begin{gathered}
U_{1}=\eta_{1} I+\left(\eta_{2}-\eta_{1}\right) e_{1} \otimes e_{1}, \quad U_{2}=\eta_{1} I+\left(\eta_{2}-\eta_{1}\right) e_{2} \otimes e_{2}, \\
U_{3}=\eta_{1} I+\left(\eta_{2}-\eta_{1}\right) e_{3} \otimes e_{3},
\end{gathered}
$$

where $\left\{e_{1}, e_{2}, e_{3}\right\}$ is an orthonormal basis for $\mathbb{R}^{3}$ and where $\eta_{1}$ and $\eta_{2}$ are positive constants such that $\eta_{1} \neq \eta_{2}$. We recall that $v \otimes w \in \mathbb{R}^{3 \times 3}$ for $v, w \in \mathbb{R}^{3}$ is the tensor product defined by $(v \otimes w) u=(w \cdot u) v$ for $u \in \mathbb{R}^{3}$, or equivalently, $(v \otimes w)_{k l}=v_{k} w_{l}$.

We note that the variants of the tetragonal phase are symmetry-related since we have for $i, j \in\{1,2,3\}, i \neq j$, that

$$
Q U_{i} Q^{T}=U_{j} \quad \text { for } Q=-I+\left(e_{i}+e_{j}\right) \otimes\left(e_{i}+e_{j}\right),
$$

where $Q=-I+\left(e_{i}+e_{j}\right) \otimes\left(e_{i}+e_{j}\right)$ is the rotation of $\pi$ radians about the axis $e_{i}+e_{j}$. We also note that

$$
Q U_{i} Q^{T}=U_{i} \quad \text { for } Q=-I+2 e_{j} \otimes e_{j}
$$

for $i, j \in\{1,2,3\}$. The symmetry group of the cube [2, 20],

$$
\mathcal{G}=\left\{Q_{1}, \ldots, Q_{24}\right\},
$$

is generated by the set of rotations $Q=-I+\left(e_{i}+e_{j}\right) \otimes\left(e_{i}+e_{j}\right)$ for $i, j \in\{1,2,3\}, i \neq$ $j$, and $Q=-I+2 e_{j} \otimes e_{j}$ for $j \in\{1,2,3\}$, so we have that

$$
\left\{Q_{i} U_{1} Q_{i}^{T}: Q_{i} \in \mathcal{G}\right\}=\left\{U_{1}, U_{2}, U_{3}\right\} .
$$

To model the cubic to tetragonal martensitic transformation, we assume that the energy density $\phi$ is minimized on the energy wells

$$
\mathcal{U}_{i} \equiv \mathrm{SO}(3) U_{i}=\left\{Q U_{i}: Q \in \mathrm{SO}(3)\right\}
$$

for $i=1,2,3$, so we may assume after rescaling the energy density that

$$
\begin{array}{cl}
\phi(F) \geq 0 \quad \forall F \in \mathbb{R}^{3 \times 3}, \\
\phi(F)=0 \quad \text { if and only if } \quad F \in \mathcal{U} \equiv \mathcal{U}_{1} \cup \mathcal{U}_{2} \cup \mathcal{U}_{3} .
\end{array}
$$

We shall also assume that the energy density $\phi$ grows quadratically away from the energy wells, that is,

$$
\phi(F) \geq \kappa\|F-\pi(F)\|^{2} \quad \forall F \in \mathbb{R}^{3 \times 3},
$$

where $\kappa>0$ is a constant and $\pi: \mathbb{R}^{3 \times 3} \rightarrow \mathcal{U}$ is a Borel measurable projection such that

$$
\|F-\pi(F)\|=\min _{G \in \mathcal{U}}\|F-G\| \quad \forall F \in \mathbb{R}^{3 \times 3} .
$$

In the above and in the following we use the matrix norm defined by

$$
\|F\|^{2} \equiv \operatorname{trace}\left(F^{T} F\right)=\sum_{i, j=1}^{3} F_{i j}^{2} \quad \forall F=\left(F_{i j}\right) \in \mathbb{R}^{3 \times 3} .
$$


The projection $\pi(F)$ exists for any $F \in \mathbb{R}^{3 \times 3}$, since $\mathcal{U}$ is compact, although the projection may not be unique. It is unique, however, if $\|F-\pi(F)\|$ is small enough $[20]$.

Two matrices $F_{0}, F_{1} \in \mathbb{R}^{3 \times 3}$ are rank-one connected $[3,20]$ if there exist $a \in \mathbb{R}^{3}$ and $n \in \mathbb{R}^{3},|n|=1$, such that

$$
F_{1}=F_{0}+a \otimes n
$$

We shall assume in what follows that $F_{0}$ and $F_{1}$ are rank-one connected as in (7) and that $F_{0} \in \mathcal{U}$ and $F_{1} \in \mathcal{U}$. We can then construct the continuous, energy-minimizing, first-order laminate $w_{\gamma}(x): \mathbb{R}^{3} \rightarrow \mathbb{R}^{3}$ with layer thickness $\gamma>0$ by

$$
w_{\gamma}(x)=\gamma w\left(\frac{x}{\gamma}\right)
$$

where

$$
w(x)=F_{0} x+\left[\int_{0}^{x \cdot n} \chi(s) d s\right] a
$$

and where $\chi(s): \mathbb{R} \rightarrow \mathbb{R}$ is the characteristic function with period 1 defined for $0<\lambda<1$ by

$$
\chi(s)= \begin{cases}0 & \text { for all } \quad 0 \leq s \leq 1-\lambda \\ 1 & \text { for all } \quad 1-\lambda<s<1\end{cases}
$$

Now by the scaling properties of $w_{\gamma}(x)$ we have that

$$
\begin{gathered}
\left|w_{\gamma}(x)-F_{\lambda} x\right|=\gamma\left|w\left(\frac{x}{\gamma}\right)-F_{\lambda}\left(\frac{x}{\gamma}\right)\right|=\gamma\left|\int_{0}^{\frac{x \cdot n}{\gamma}}[\chi(s)-\lambda] d s a\right| \\
\leq \lambda(1-\lambda)|a| \gamma
\end{gathered}
$$

where

$$
F_{\lambda}=(1-\lambda) F_{0}+\lambda F_{1}=F_{0}+\lambda a \otimes n
$$

We also have

$$
\nabla w_{\gamma}(x)=F_{0}+\chi\left(\frac{x \cdot n}{\gamma}\right) a \otimes n \quad \text { for almost all } x \in \Omega,
$$

So

$$
\nabla w_{\gamma}(x)=\left\{\begin{array}{lll}
F_{0} & \text { if } j \gamma<x \cdot n<(j+1-\lambda) \gamma & \text { for some } j \in \mathbb{Z} \\
F_{1} & \text { if }(j+1-\lambda) \gamma<x \cdot n<(j+1) \gamma & \text { for some } j \in \mathbb{Z}
\end{array}\right.
$$

Hence, it follows from (5) and (10) that

$$
\int_{\Omega} \phi\left(\nabla w_{\gamma}(x)\right) d x=0
$$

since $F_{0}, F_{1} \in \mathcal{U}$. We see by (9) that the deformations $w_{\gamma}(x)$ converge uniformly to $F_{\lambda} x$ as $\gamma \rightarrow 0$, but by (10) the deformation gradients oscillate between $F_{0}$ in layers of thickness $(1-\lambda) \gamma$ and $F_{1}$ in layers of thickness $\lambda \gamma$. 
We consider in this paper the approximation of the energy-minimizing microstructure subject to the boundary conditions

$$
y(x)=F_{\lambda} x=\left[(1-\lambda) F_{0}+\lambda F_{1}\right] x \quad \forall x \in \partial \Omega
$$

for $0<\lambda<1$ and $F_{0}, F_{1} \in \mathcal{U}$. Thus, we will consider the minimization of the energy (1) with respect to the set of admissible deformations given by

$$
W_{F_{\lambda}}^{1, \infty}\left(\Omega ; \mathbb{R}^{3}\right) \equiv\left\{y \in W^{1, \infty}\left(\Omega ; \mathbb{R}^{3}\right): y(x)=F_{\lambda} x \text { for } x \in \partial \Omega\right\} .
$$

The following two lemmas $[2,3,20]$ show that for the cubic to tetragonal transformation (2) each $F_{0} \in \mathcal{U}_{i}$ is not rank-one connected to any $F_{1} \in \mathcal{U}_{i}$ with $F_{0} \neq F_{1}$, but that every $F_{0} \in \mathcal{U}_{i}$ is rank-one connected to two distinct $F_{1} \in \mathcal{U}_{j}$ for all $j \neq i$, $j \in\{1,2,3\}$.

LEMma 1. If $F_{0} \in \mathcal{U}_{i}$ for some $i \in\{1,2,3\}$, then there does not exist $F_{1} \in \mathcal{U}_{i}$ with $F_{0} \neq F_{1}$, such that $F_{0}$ and $F_{1}$ are rank-one connected.

LEMMA 2. If $F_{0} \in \mathcal{U}_{i}$ for some $i \in\{1,2,3\}$, then for any $j \neq i, j \in\{1,2,3\}$, there exist two distinct $F_{1} \in \mathcal{U}_{j}$ such that $F_{0}$ and $F_{1}$ are rank-one connected. Further, if $F_{0} \in \mathcal{U}_{i}$ and $F_{1} \in \mathcal{U}_{j}$ are rank-one connected so that

$$
F_{1}=F_{0}+a \otimes n
$$

for $a \in \mathbb{R}^{3}$ and $n \in \mathbb{R}^{3},|n|=1$, then

$$
n \in\left\{ \pm \frac{1}{\sqrt{2}}\left(e_{i}+e_{j}\right), \pm \frac{1}{\sqrt{2}}\left(e_{i}-e_{j}\right)\right\} .
$$

We note that $n$ and $-n$ give the same rank-one connections for $F_{0}$ and $F_{1}$ in (11) since

$$
F_{1}=F_{0}+a \otimes n=F_{0}+(-a) \otimes(-n) .
$$

The planes with normal vectors $n$ given by (12) are known as the "twin planes" in the crystallographical literature. The continuous, energy-minimizing, first-order laminate $w_{\gamma}(x)$ given by $(8)$ has distinct constant gradients in layers which are parallel to the twin planes. It can be easily shown by Lemma 2 that there exists a continuous energy-minimizing deformation with a distinct constant gradient on each side of a smooth interface if and only if the interface is a twin plane [2, 3, 20].

3. Estimates for directional derivatives in the twin plane. We recall that

$$
F_{\lambda}=(1-\lambda) F_{0}+\lambda F_{1},
$$

where $0<\lambda<1$ and where $F_{0} \in \mathcal{U}$ and $F_{1} \in \mathcal{U}$ are rank-one connected so that

$$
F_{1}=F_{0}+a \otimes n
$$

and we recall that

$$
W_{F_{\lambda}}^{1, \infty}\left(\Omega ; \mathbb{R}^{3}\right) \equiv\left\{y \in W^{1, \infty}\left(\Omega ; \mathbb{R}^{3}\right): y(x)=F_{\lambda} x \text { for } x \in \partial \Omega\right\} .
$$

It follows from Lemma 2 that $F_{0} \in \mathcal{U}_{k}$ and $F_{1} \in \mathcal{U}_{l}$, for some $k, l \in\{1,2,3\}$ such that $k \neq l$. 
Lemma 3. For any $y \in W_{F_{\lambda}}^{1, \infty}\left(\Omega ; \mathbb{R}^{3}\right)$, we have

$$
\int_{\Omega}\|\nabla y(x)-\pi(\nabla y(x))\|^{2} d x \leq \kappa^{-1} \mathcal{E}(y) .
$$

Proof. The inequality follows directly from the quadratic growth rate of the energy density $\phi$ away from the energy wells (6).

The next lemma gives an estimate for the convergence of the directional derivatives in the twin plane for energy-minimizing sequences of deformations. In the following, $C$ will denote a generic positive constant which is independent of $y \in$ $W_{F_{\lambda}}^{1, \infty}\left(\Omega ; \mathbb{R}^{3}\right)$.

Lemma 4. If $w \in \mathbb{R}^{3}$ satisfies $w \cdot n=0$, then there exists a positive constant $C$ such that

$$
\int_{\Omega}\left|\left[\pi(\nabla y(x))-F_{\lambda}\right] w\right|^{2} d x \leq C \mathcal{E}(y)^{1 / 2} \quad \forall y \in W_{F_{\lambda}}^{1, \infty}\left(\Omega ; \mathbb{R}^{3}\right) .
$$

Proof. We may assume without loss of generality, by the symmetry relations (3) and (4) and by Lemma 2 , that $F_{0} \in \mathcal{U}_{1}, F_{1} \in \mathcal{U}_{2}$,

$$
n=\frac{1}{\sqrt{2}}\left(e_{1}+e_{2}\right)
$$

and

$$
F_{1}=F_{0}+a \otimes n
$$

for some $a \in \mathbb{R}^{3}$. We define

$$
w_{1}=e_{1}-e_{2}+e_{3} \quad \text { and } \quad w_{2}=e_{1}-e_{2}-e_{3} .
$$

It is easy to check that

$$
\left|U_{i} w_{j}\right|=\sqrt{2 \eta_{1}^{2}+\eta_{2}^{2}}
$$

for all $i \in\{1,2,3\}$ and $j \in\{1,2\}$. We also have for any $i \in\{1,2,3\}$ that

$$
\left|Q U_{i} \tilde{w}\right|=\left|U_{i} \tilde{w}\right| \quad \forall Q \in \mathrm{SO}(3), \forall \tilde{w} \in \mathbb{R}^{3} .
$$

We can thus conclude by (16) and (17) that

$$
\left|U w_{j}\right|=\sqrt{2 \eta_{1}^{2}+\eta_{2}^{2}} \quad \forall U \in \mathcal{U}, \forall j \in\{1,2\}
$$

since $\mathcal{U}=\mathcal{U}_{1} \cup \mathcal{U}_{2} \cup \mathcal{U}_{3}$ and $\mathcal{U}_{i}=\mathrm{SO}(3) U_{i}$.

Since $\left\{e_{1}, e_{2}, e_{3}\right\}$ is an orthonormal basis for $\mathbb{R}^{3}$, we have that $w_{1} \cdot n=w_{2} \cdot n=0$. Thus, it follows that we have for $j \in\{1,2\}$ by the rank-one connection (14) that

$$
F_{1} w_{j}=F_{0} w_{j}=F_{\lambda} w_{j}
$$

SO

$$
\left|F_{1} w_{j}\right|=\left|F_{0} w_{j}\right|=\left|F_{\lambda} w_{j}\right| .
$$


Since $\pi(F) \in \mathcal{U}$ for all $F \in \mathbb{R}^{3 \times 3}$ and $F_{0}, F_{1} \in \mathcal{U}$ we can obtain from (18) and (20) that for $j \in\{1,2\}$ we have

$$
\left|\pi(F) w_{j}\right|=\left|F_{\lambda} w_{j}\right| \quad \forall F \in \mathbb{R}^{3 \times 3} .
$$

We have that

$$
F_{\lambda}=\frac{1}{\operatorname{meas}(\Omega)} \int_{\Omega} \nabla y(x) d x
$$

since $y(x)=F_{\lambda} x$ for $x \in \partial \Omega$. It then follows from (21), (22), the Cauchy-Schwarz inequality, and Lemma 3 that for $j \in\{1,2\}$

$$
\begin{aligned}
\int_{\Omega}\left|\left[\pi(\nabla y(x))-F_{\lambda}\right] w_{j}\right|^{2} d x \\
\quad=2 F_{\lambda} w_{j} \cdot \int_{\Omega}\left[F_{\lambda}-\pi(\nabla y(x))\right] w_{j} d x \\
=2 F_{\lambda} w_{j} \cdot \int_{\Omega}[\nabla y(x)-\pi(\nabla y(x))] w_{j} d x \\
\leq C \int_{\Omega}\|\nabla y(x)-\pi(\nabla y(x))\| d x \\
\leq C\left[\int_{\Omega}\|\nabla y(x)-\pi(\nabla y(x))\|^{2} d x\right]^{1 / 2} \\
\leq C \mathcal{E}(y)^{1 / 2}
\end{aligned}
$$

Finally, since $\left\{w_{1}, w_{2}\right\}$ is a basis for the two-dimensional subspace defined by $w \cdot n=0$, we have that (13) holds for any $w \in \mathbb{R}^{3}$ such that $w \cdot n=0$.

The following theorem giving bounds on the directional derivatives orthogonal to $n$ is a consequence of the above two lemmas and the triangle inequality and will play a key role in establishing all of the other bounds.

THEOREM 1. If $w \in \mathbb{R}^{3}$ satisfies $w \cdot n=0$, then there exists a positive constant C such that

$$
\int_{\Omega}\left|\left[\nabla y(x)-F_{\lambda}\right] w\right|^{2} d x \leq C\left[\mathcal{E}(y)^{1 / 2}+\mathcal{E}(y)\right] \quad \forall y \in W_{F_{\lambda}}^{1, \infty}\left(\Omega ; \mathbb{R}^{3}\right) .
$$

4. Estimates for deformations and deformation gradients. We now give bounds on the convergence of energy-minimizing sequences of deformations in $W_{F_{\lambda}}^{1, \infty}\left(\Omega ; \mathbb{R}^{3}\right)$ to the homogeneous deformation $F_{\lambda} x$.

THEOREM 2. There exists a positive constant $C$ such that

$$
\int_{\Omega}\left|y(x)-F_{\lambda} x\right|^{2} d x \leq C\left[\mathcal{E}(y)^{1 / 2}+\mathcal{E}(y)\right] \quad \forall y \in W_{F_{\lambda}}^{1, \infty}\left(\Omega ; \mathbb{R}^{3}\right) .
$$

Proof. If $y \in W_{F_{\lambda}}^{1, \infty}\left(\Omega ; \mathbb{R}^{3}\right)$, then $y(x)-F_{\lambda} x=0$ for all $x \in \partial \Omega$. Thus, for any $w \in \mathbb{R}^{3}$ there exists a positive constant $C$ by the Poincaré inequality $[21,31]$ so that

$$
\int_{\Omega}\left|y(x)-F_{\lambda} x\right|^{2} d x \leq C \int_{\Omega}\left|\left[\nabla y(x)-F_{\lambda}\right] w\right|^{2} d x \quad \forall y \in W_{F_{\lambda}}^{1, \infty}\left(\Omega ; \mathbb{R}^{3}\right) .
$$

Our assertion follows from Theorem 1 , with $w \in \mathbb{R}^{3}$ so chosen that $w \cdot n=0$. 
Our next theorem gives an estimate for the weak convergence of the gradients of energy-minimizing sequences of deformations. The proof follows from Lemma 3 and Theorem 2.

THEOREM 3. For any Lipschitz domain $\omega \subset \Omega$, there exists a positive constant $C$ such that

$$
\left\|\int_{\omega}\left[\nabla y(x)-F_{\lambda}\right] d x\right\| \leq C\left[\mathcal{E}(y)^{1 / 8}+\mathcal{E}(y)^{1 / 2}\right] \quad \forall y \in W_{F_{\lambda}}^{1, \infty}\left(\Omega ; \mathbb{R}^{3}\right) .
$$

Proof. If follows from the divergence theorem and the Cauchy-Schwarz inequality that

$$
\begin{gathered}
\left\|\int_{\omega}\left[\nabla y(x)-F_{\lambda}\right] d x\right\|=\left\|\int_{\partial \omega}\left[y(x)-F_{\lambda} x\right] \otimes \nu d S\right\| \\
\leq \int_{\partial \omega}\left|y(x)-F_{\lambda} x\right| d S \\
\quad \leq \operatorname{meas}_{2}(\partial \omega)^{1 / 2}\left(\int_{\partial \omega}\left|y(x)-F_{\lambda} x\right|^{2} d S\right)^{1 / 2}
\end{gathered}
$$

where $\nu$ is the unit exterior normal to $\partial \omega$ and where $\operatorname{meas}_{2}(\partial \omega)$ is the surface area of $\partial \omega$. We can obtain by the trace theorem $[1,31]$ that

$$
\begin{aligned}
\int_{\partial \omega}\left|y(x)-F_{\lambda} x\right|^{2} d S & \\
\leq C & {\left[\int_{\omega}\left|y(x)-F_{\lambda} x\right|^{2} d x+\int_{\omega}|\nabla| y(x)-\left.F_{\lambda} x\right|^{2} \mid d x\right] } \\
\leq C & {\left[\int_{\omega}\left|y(x)-F_{\lambda} x\right|^{2} d x+\int_{\omega}\left|y(x)-F_{\lambda} x\right| \cdot\left\|\nabla\left[y(x)-F_{\lambda} x\right]\right\| d x\right] } \\
\leq C & {\left[\int_{\omega}\left|y(x)-F_{\lambda} x\right|^{2} d x\right.} \\
+ & \left.\left(\int_{\omega}\left|y(x)-F_{\lambda} x\right|^{2} d x\right)^{1 / 2}\left(\int_{\omega}\left\|\nabla\left[y(x)-F_{\lambda} x\right]\right\|^{2} d x\right)^{1 / 2}\right] .
\end{aligned}
$$

We have by the triangle inequality and Lemma 3 that

$$
\begin{aligned}
& \left(\int_{\omega}\left\|\nabla y(x)-F_{\lambda}\right\|^{2} d x\right)^{1 / 2} \\
& \quad \leq\left(\int_{\omega}\|\nabla y(x)-\pi(\nabla y(x))\|^{2} d x\right)^{1 / 2}+\left(\int_{\omega}\left\|\pi(\nabla y(x))-F_{\lambda}\right\|^{2} d x\right)^{1 / 2} \\
& \quad \leq \kappa^{-1 / 2} \mathcal{E}(y)^{1 / 2}+2\left(2 \eta_{1}^{2}+\eta_{2}^{2}\right)^{1 / 2} \operatorname{meas}(\omega)^{1 / 2} .
\end{aligned}
$$

It follows by using Theorem 2 and (25) in (24) that

$$
\begin{aligned}
& \int_{\partial \omega} \mid y(x)-\left.F_{\lambda} x\right|^{2} d S \leq C\left[\mathcal{E}(y)^{1 / 4}+\mathcal{E}(y)^{1 / 2}+\mathcal{E}(y)\right] \\
& \leq C\left[\mathcal{E}(y)^{1 / 4}+\mathcal{E}(y)\right] \quad \forall y \in W_{F_{\lambda}}^{1, \infty}\left(\Omega ; \mathbb{R}^{3}\right),
\end{aligned}
$$

since $\beta^{1 / 2} \leq \beta+\beta^{1 / 4}$ for $\beta>0$. We finally obtain the result of Theorem 3 by substituting (26) into (23). 
We recall that $F_{0} \in \mathcal{U}_{k}, F_{1} \in \mathcal{U}_{l}$ for $k, l \in\{1,2,3\}$ such that $k \neq l$. We define the projection operator $\pi_{k l}: \mathbb{R}^{3 \times 3} \rightarrow \mathcal{U}_{k} \cup \mathcal{U}_{l}$ by

$$
\left\|F-\pi_{k l}(F)\right\|=\min _{G \in \mathcal{U}_{k} \cup \mathcal{U}_{l}}\|F-G\| \quad \forall F \in \mathbb{R}^{3 \times 3} .
$$

We also define the operators $R: \mathbb{R}^{3 \times 3} \rightarrow \mathrm{SO}(3)$ and $\Pi: \mathbb{R}^{3 \times 3} \rightarrow\left\{F_{0}, F_{1}\right\}$ by the relation

$$
\pi_{k l}(F)=R(F) \Pi(F) \quad \forall F \in \mathbb{R}^{3 \times 3} .
$$

The proof of the following lemma shows that the measure of the set of points in which the gradient of energy-minimizing sequences of deformations is near $\mathcal{U}_{m}$ for $m \in\{1,2,3\}$ such that $m \neq k$ and $m \neq l$ converges to zero.

Lemma 5. If $F_{0} \in \mathcal{U}_{k}$ and $F_{1} \in \mathcal{U}_{l}$ for $k, l \in\{1,2,3\}, k \neq l$, then there exists a positive constant $C$ such that

$$
\int_{\Omega}\left\|\nabla y(x)-\pi_{k l}(\nabla y(x))\right\|^{2} d x \leq C\left[\mathcal{E}(y)^{1 / 2}+\mathcal{E}(y)\right] \quad \forall y \in W_{F_{\lambda}}^{1, \infty}\left(\Omega ; \mathbb{R}^{3}\right) .
$$

Proof. We can again assume without loss of generality that $F_{0} \in \mathcal{U}_{1}, F_{1} \in \mathcal{U}_{2}$,

$$
n=\frac{1}{\sqrt{2}}\left(e_{1}+e_{2}\right)
$$

and

$$
F_{1}=F_{0}+a \otimes n
$$

for some $a \in \mathbb{R}^{3}$. We have for any $Q \in \mathrm{SO}(3)$ that

$$
Q U_{3} e_{3} \neq F_{\lambda} e_{3}
$$

since

$$
\left|Q U_{3} e_{3}\right|=\left|U_{3} e_{3}\right|=\eta_{2} \quad \text { and } \quad\left|F_{\lambda} e_{3}\right|=\left|F_{0} e_{3}\right|=\left|U_{1} e_{3}\right|=\eta_{1} .
$$

Thus, we have by the triangle inequality that

$$
\inf _{F \in \mathcal{U}_{3}}\left|F_{\lambda} e_{3}-F e_{3}\right| \geq\left|\eta_{2}-\eta_{1}\right|
$$

Denoting

$$
\Omega_{3} \equiv\left\{x \in \Omega: \pi(\nabla y(x)) \in \mathcal{U}_{3}\right\}
$$

we then obtain by Lemma 4 that

$$
\begin{aligned}
& \operatorname{meas}\left(\Omega_{3}\right)=\int_{\Omega_{3}} d x \\
& \quad \leq\left|\eta_{2}-\eta_{1}\right|^{-2} \int_{\Omega_{3}}\left|\left[\pi(\nabla y(x))-F_{\lambda}\right] e_{3}\right|^{2} d x \\
& \quad \leq C \mathcal{E}(y)^{1 / 2}
\end{aligned}
$$


since $e_{3} \cdot n=0$. Consequently, we have by Lemma 3 that (recall that we have assumed that $k=1$ and $l=2$ )

$$
\begin{aligned}
\int_{\Omega} \| & \nabla y(x)-\pi_{12}(\nabla y(x)) \|^{2} d x \\
& \leq 2 \int_{\Omega}\|\nabla y(x)-\pi(\nabla y(x))\|^{2} d x+2 \int_{\Omega}\left\|\pi(\nabla y(x))-\pi_{12}(\nabla y(x))\right\|^{2} d x \\
& \leq 2 \int_{\Omega}\|\nabla y(x)-\pi(\nabla y(x))\|^{2} d x+8\left(2 \eta_{1}^{2}+\eta_{2}^{2}\right) \operatorname{meas}\left(\Omega_{3}\right) \\
& \leq C\left[\mathcal{E}(y)^{1 / 2}+\mathcal{E}(y)\right] .
\end{aligned}
$$

The following theorem gives estimates for the convergence of gradients of energyminimizing sequences of deformations to the set $\left\{F_{0}, F_{1}\right\}$.

THEOREM 4. There exists a positive constant $C$ such that

$$
\int_{\Omega}\|\nabla y(x)-\Pi(\nabla y(x))\|^{2} d x \leq C\left[\mathcal{E}(y)^{1 / 2}+\mathcal{E}(y)\right] \quad \forall y \in W_{F_{\lambda}}^{1, \infty}\left(\Omega ; \mathbb{R}^{3}\right) .
$$

Proof. We again assume without loss of generality that $F_{0} \in \mathcal{U}_{1}, F_{1} \in \mathcal{U}_{2}$, and

$$
n=\frac{1}{\sqrt{2}}\left(e_{1}+e_{2}\right)
$$

We define as in (15)

$$
w_{1}=e_{1}-e_{2}+e_{3} \quad \text { and } \quad w_{2}=e_{1}-e_{2}-e_{3} .
$$

Since $w_{j} \cdot n=0$ for $j=1,2$, we have by (19) that

$$
\Pi(F) w_{j}=F_{1} w_{j}=F_{0} w_{j}=F_{\lambda} w_{j} \quad \forall F \in \mathbb{R}^{3 \times 3} .
$$

Thus, it follows from (27) with $k=1$ and $l=2$ that

$$
\begin{aligned}
& {[R(F)-I] F_{0} w_{j}=[R(F)-I] \Pi(F) w_{j}=\left[\pi_{12}(F)-F_{\lambda}\right] w_{j}} \\
& \quad=\left[\pi_{12}(F)-\pi(F)\right] w_{j}+\left[\pi(F)-F_{\lambda}\right] w_{j} \quad \forall F \in \mathbb{R}^{3 \times 3} .
\end{aligned}
$$

We can then apply the triangle inequality to (29) with $F=\nabla y(x)$ and estimate the two terms by (28) and Lemma 4 to obtain

$$
\begin{aligned}
& \int_{\Omega}\left|[R(\nabla y(x))-I] F_{0} w_{j}\right|^{2} d x \\
& \quad \leq 2 \int_{\Omega}\left|\left[\pi_{12}(\nabla y(x))-\pi(\nabla y(x))\right] w_{j}\right|^{2} d x+2 \int_{\Omega}\left|\left[\pi(\nabla y(x))-F_{\lambda}\right] w_{j}\right|^{2} d x \\
& \quad \leq C \mathcal{E}(y)^{1 / 2} \quad \text { for } j=1,2 .
\end{aligned}
$$

We have for

$$
m=F_{0} w_{1} \times F_{0} w_{2}
$$

that

$$
Q m=Q F_{0} w_{1} \times Q F_{0} w_{2} \quad \forall Q \in \mathrm{SO}(3) .
$$


Thus, we have the identity

$$
\begin{aligned}
& {[R(F)-I] m=\left\{R(F) F_{0} w_{1} \times R(F) F_{0} w_{2}\right\}-\left\{F_{0} w_{1} \times F_{0} w_{2}\right\}} \\
& \quad=\left\{[R(F)-I] F_{0} w_{1} \times R(F) F_{0} w_{2}\right\}-\left\{F_{0} w_{1} \times[I-R(F)] F_{0} w_{2}\right\}
\end{aligned}
$$

for all $F \in \mathbb{R}^{3 \times 3}$. We can then obtain from using the above identity with $F=\nabla y(x)$ and the estimates (30) that

$$
\int_{\Omega}|[R(\nabla y(x))-I] m|^{2} d x \leq C \mathcal{E}(y)^{1 / 2} .
$$

Now $\left\{F_{0} w_{1}, F_{0} w_{2}, m\right\}$ is a basis for $\mathbb{R}^{3}$, so we have from (30) and (31) that

$$
\int_{\Omega}\|[R(\nabla y(x))-I]\|^{2} d x \leq C\left[\mathcal{E}(y)^{1 / 2}+\mathcal{E}(y)\right] \quad \forall y \in W_{F_{\lambda}}^{1, \infty}\left(\Omega ; \mathbb{R}^{3}\right) .
$$

We can then prove Theorem 4 by applying the triangle inequality to the identity

$$
\begin{aligned}
F-\Pi(F) & =\left[F-\pi_{12}(F)\right]+\left[\pi_{12}(F)-\Pi(F)\right] \\
& =\left[F-\pi_{12}(F)\right]+[R(F)-I] \Pi(F) \quad \forall F \in \mathbb{R}^{3 \times 3}
\end{aligned}
$$

with $F=\nabla y(x)$ and by estimating the two terms by Lemma 5 and (32).

5. Estimates for the volume fractions and nonlinear integrals of the deformation gradients. For any subset $\omega \subset \Omega, \rho>0$, and $y \in W_{F_{\lambda}}^{1, \infty}\left(\Omega ; \mathbb{R}^{3}\right)$, we define the sets

$$
\begin{aligned}
& \omega_{\rho}^{0}(y)=\left\{x \in \omega: \Pi(\nabla y(x))=F_{0} \text { and }\left\|F_{0}-\nabla y(x)\right\|<\rho\right\}, \\
& \omega_{\rho}^{1}(y)=\left\{x \in \omega: \Pi(\nabla y(x))=F_{1} \text { and }\left\|F_{1}-\nabla y(x)\right\|<\rho\right\} .
\end{aligned}
$$

The following theorem states that for any Lipschitz domain $\omega \subset \Omega$ and for any energyminimizing sequence $\left\{y_{k}\right\}$ in $W_{F_{\lambda}}^{1, \infty}\left(\Omega ; \mathbb{R}^{3}\right)$ the volume fraction that $\nabla y_{k}$ is near $F_{0}$ converges to $1-\lambda$ and the volume fraction that $\nabla y_{k}$ is near $F_{1}$ converges to $\lambda$. We note that the uniqueness of the Young measure for the minimization of the energy (1) for the cubic to tetragonal transformation with respect to the set $W_{F_{\lambda}}^{1, \infty}\left(\Omega ; \mathbb{R}^{3}\right)[3]$ is a consequence of the following theorem [20].

THEOREM 5. For any Lipschitz domain $\omega \subset \Omega$ and any $\rho>0$, there exists a positive constant $C$ such that

$$
\left|\frac{\operatorname{meas} \omega_{\rho}^{0}(y)}{\operatorname{meas} \omega}-(1-\lambda)\right|+\left|\frac{\operatorname{meas} \omega_{\rho}^{1}(y)}{\operatorname{meas} \omega}-\lambda\right| \leq C\left[\mathcal{E}(y)^{1 / 8}+\mathcal{E}(y)^{1 / 2}\right]
$$

for all $y \in W_{F_{\lambda}}^{1, \infty}\left(\Omega ; \mathbb{R}^{3}\right)$.

Proof. It follows from the definition of $\omega_{\rho}^{0} \equiv \omega_{\rho}^{0}(y)$ and $\omega_{\rho}^{1} \equiv \omega_{\rho}^{1}(y)$ that

$$
\begin{aligned}
& {\left[\operatorname{meas} \omega_{\rho}^{0}-(1-\lambda) \operatorname{meas} \omega\right] F_{0}+\left[\operatorname{meas} \omega_{\rho}^{1}-\lambda \text { meas } \omega\right] F_{1}} \\
& \quad=\int_{\omega}\left[\Pi(\nabla y(x))-F_{\lambda}\right] d x-\int_{\omega-\left\{\omega_{\rho}^{0} \cup \omega_{\rho}^{1}\right\}} \Pi(\nabla y(x)) d x .
\end{aligned}
$$

We have by the triangle inequality, the Cauchy-Schwarz inequality, Theorem 4, and 
Theorem 3 that

$$
\begin{aligned}
& \left\|\int_{\omega}\left[\Pi(\nabla y(x))-F_{\lambda}\right] d x\right\| \\
& \leq\left\|\int_{\omega}[\Pi(\nabla y(x))-\nabla y(x)] d x\right\|+\left\|\int_{\omega}\left[\nabla y(x)-F_{\lambda}\right] d x\right\| \\
& \quad \leq \operatorname{meas}(\omega)^{1 / 2}\left[\int_{\omega}\|\Pi(\nabla y(x))-\nabla y(x)\|^{2} d x\right]^{1 / 2}+\left\|\int_{\omega}\left[\nabla y(x)-F_{\lambda}\right] d x\right\| \\
& \leq C\left[\mathcal{E}(y)^{1 / 8}+\mathcal{E}(y)^{1 / 2}\right] \quad \forall y \in W_{F_{\lambda}}^{1, \infty}\left(\Omega ; \mathbb{R}^{3}\right) .
\end{aligned}
$$

Next, we have by the definition of $\omega_{\rho}^{0}$ and $\omega_{\rho}^{1}$ that

$$
\operatorname{meas}\left(\omega-\left\{\omega_{\rho}^{0} \cup \omega_{\rho}^{1}\right\}\right) \leq \frac{1}{\rho} \int_{\omega-\left\{\omega_{\rho}^{0} \cup \omega_{\rho}^{1}\right\}}\|\Pi(\nabla y(x))-\nabla y(x)\| d x
$$

Since $\|\Pi(F)\|=\sqrt{2 \eta_{1}^{2}+\eta_{2}^{2}}$ for all $F \in \mathbb{R}^{3 \times 3}$, we can conclude from (34) and Theorem 4 that

$$
\begin{aligned}
& \left\|\int_{\omega-\left\{\omega_{\rho}^{0} \cup \omega_{\rho}^{1}\right\}} \Pi(\nabla y(x)) d x\right\| \leq C \operatorname{meas}\left(\omega-\left\{\omega_{\rho}^{0} \cup \omega_{\rho}^{1}\right\}\right) \\
& \leq \frac{C}{\rho} \int_{\omega-\left\{\omega_{\rho}^{0} \cup \omega_{\rho}^{1}\right\}}\|\Pi(\nabla y(x))-\nabla y(x)\| d x \\
& \leq \frac{C(\operatorname{meas} \omega)^{1 / 2}}{\rho}\left[\int_{\omega}\|\Pi(\nabla y(x))-\nabla y(x)\|^{2} d x\right]^{1 / 2} \\
& \leq C\left[\mathcal{E}(y)^{1 / 4}+\mathcal{E}(y)^{1 / 2}\right] .
\end{aligned}
$$

Hence, we have from (33) and (35) that

$$
\begin{gathered}
\left\|\left[\operatorname{meas} \omega_{\rho}^{0}-(1-\lambda) \operatorname{meas} \omega\right] F_{0}+\left[\operatorname{meas} \omega_{\rho}^{1}-\lambda \operatorname{meas} \omega\right] F_{1}\right\| \\
\leq C\left[\mathcal{E}(y)^{1 / 8}+\mathcal{E}(y)^{1 / 2}\right]
\end{gathered}
$$

Our assertion now follows from the linear independence of $F_{0}$ and $F_{1}$.

For linear transformations $\mathcal{L}: \mathbb{R}^{3 \times 3} \rightarrow \mathbb{R}$ we define the operator norm

$$
\|\mathcal{L}\|=\max _{\|A\|=1}|\mathcal{L}(A)|
$$

so for Lipschitz functions $g(A): \mathbb{R}^{3 \times 3} \rightarrow \mathbb{R}$ we can define the function norm

$$
\left\|\frac{\partial g}{\partial A}\right\|_{L^{\infty}}=\operatorname{ess} \sup _{B \in \mathbf{R}^{3 \times 3}}\left\|\frac{\partial g}{\partial A}(B)\right\| .
$$

We obtain estimates for the Sobolev space $\mathcal{V}$ of measurable functions $f(x, A): \Omega \times$ $\mathbb{R}^{3 \times 3} \rightarrow \mathbb{R}$ such that

$$
\|f\|_{\mathcal{V}}^{2}=\int_{\Omega}\left[\left\|\frac{\partial f}{\partial A}(x, \cdot)\right\|_{L^{\infty}}^{2}+|\nabla G(x) n|^{2}+G(x)^{2}\right] d x<\infty
$$


where

$$
G(x)=f\left(x, F_{1}\right)-f\left(x, F_{0}\right) .
$$

We observe that if $f(x, A) \in \mathcal{V}$, then $f(x, A)$ is Lipschitz continuous as a function of $A \in \mathbb{R}^{3 \times 3}$ for almost all $x \in \Omega$.

THEOREM 6 . There exists a positive constant $C$ such that

$$
\begin{aligned}
& \left|\int_{\Omega}\left\{f(x, \nabla y(x))-\left[(1-\lambda) f\left(x, F_{0}\right)+\lambda f\left(x, F_{1}\right)\right]\right\} d x\right| \\
& \quad \leq C\|f\|_{\mathcal{V}}\left[\mathcal{E}(y)^{1 / 4}+\mathcal{E}(y)^{1 / 2}\right] \quad \forall f \in \mathcal{V}, \forall y \in W_{F_{\lambda}}^{1, \infty}\left(\Omega ; \mathbb{R}^{3}\right) .
\end{aligned}
$$

Proof. It is convenient to divide the integral (37) into two terms by

$$
\begin{aligned}
\int_{\Omega}\left\{f(x, \nabla y(x))-\left[(1-\lambda) f\left(x, F_{0}\right)+\lambda f\left(x, F_{1}\right)\right]\right\} d x \\
=\int_{\Omega}[f(x, \nabla y(x))-f(x, \Pi(\nabla y(x)))] d x \\
\quad \quad+\int_{\Omega}\left\{f(x, \Pi(\nabla y(x)))-\left[(1-\lambda) f\left(x, F_{0}\right)+\lambda f\left(x, F_{1}\right)\right]\right\} d x \\
=\mathcal{J}_{1}+\mathcal{J}_{2} .
\end{aligned}
$$

The term $\mathcal{J}_{1}$ can be estimated by Theorem 4 as follows:

$$
\begin{aligned}
\left|\mathcal{J}_{1}\right| & \leq \int_{\Omega}\left\|\frac{\partial f}{\partial A}(x, \cdot)\right\|_{L^{\infty}}\|\nabla y(x)-\Pi(\nabla y(x))\| d x \\
& \leq\left[\int_{\Omega}\left\|\frac{\partial f}{\partial A}(x, \cdot)\right\|_{L^{\infty}}^{2} d x\right]^{1 / 2}\left[\int_{\Omega}\|\nabla y(x)-\Pi(\nabla y(x))\|^{2} d x\right]^{1 / 2} \\
& \leq C\left[\int_{\Omega}\left\|\frac{\partial f}{\partial A}(x, \cdot)\right\|_{L^{\infty}}^{2} d x\right]^{1 / 2}\left[\mathcal{E}(y)^{1 / 4}+\mathcal{E}(y)^{1 / 2}\right] .
\end{aligned}
$$

We have for $G(x)=f\left(x, F_{1}\right)-f\left(x, F_{0}\right)$ the identity

$$
\begin{aligned}
f(x, & \Pi(A))-\left[(1-\lambda) f\left(x, F_{0}\right)+\lambda f\left(x, F_{1}\right)\right] \\
& =\frac{1}{|a|^{2}}\left\{a \cdot\left[\Pi(A)-F_{\lambda}\right] n\right\}\left[f\left(x, F_{1}\right)-f\left(x, F_{0}\right)\right] \\
& =\frac{1}{|a|^{2}}\left\{a \cdot\left[\Pi(A)-F_{\lambda}\right] n\right\} G(x),
\end{aligned}
$$

so it follows from (39) and integration by parts that

$$
\begin{aligned}
\mathcal{J}_{2}= & \int_{\Omega}\left\{f(x, \Pi(\nabla y(x)))-\left[(1-\lambda) f\left(x, F_{0}\right)+\lambda f\left(x, F_{1}\right)\right]\right\} d x \\
& =\frac{1}{|a|^{2}} \int_{\Omega}\left\{a \cdot\left[\Pi(\nabla y(x))-F_{\lambda}\right] n\right\} G(x) d x \\
& =\frac{1}{|a|^{2}} \int_{\Omega}\{a \cdot[\Pi(\nabla y(x))-\nabla y(x)] n\} G(x) d x
\end{aligned}
$$




$$
\begin{gathered}
+\frac{1}{|a|^{2}} \int_{\Omega}\left\{a \cdot\left[\nabla y(x)-F_{\lambda}\right] n\right\} G(x) d x \\
=\frac{1}{|a|^{2}} \int_{\Omega}\{a \cdot[\Pi(\nabla y(x))-\nabla y(x)] n\} G(x) d x \\
\quad-\frac{1}{|a|^{2}} \int_{\Omega}\left\{a \cdot\left[y(x)-F_{\lambda} x\right]\right\}\{\nabla G(x) \cdot n\} d x .
\end{gathered}
$$

We can thus conclude from the Cauchy-Schwarz inequality, Lemma 3, and Theorem 4 that

$$
\left|\mathcal{J}_{2}\right| \leq C\left\{\int_{\Omega}|\nabla G(x) \cdot n|^{2} d x+\int_{\Omega} G(x)^{2} d x\right\}^{1 / 2}\left[\mathcal{E}(y)^{1 / 4}+\mathcal{E}(y)^{1 / 2}\right] .
$$

We can then obtain the result (37) of Theorem 6 from (38) and (40).

6. Finite element approximations. We now define the properties of the conforming finite element spaces required for our numerical analysis of microstructure. We assume that $\tau_{h}$ for $0<h<h_{0}$, where $h_{0}$ is a positive constant, is a family of decompositions of $\Omega$ into polyhedra $\{K\}$ such that $[9,28]$ :

1. $\bar{\Omega}=\cup_{K \in \tau_{h}} K$;

2. interior $K_{1} \cap$ interior $K_{2}=\emptyset$ if $K_{1} \neq K_{2}$ for $K_{1}, K_{2} \in \tau_{h}$;

3. if $S=K_{1} \cap K_{2} \neq \emptyset$ for $K_{1} \neq K_{2}, K_{1}, K_{2} \in \tau_{h}$, then $S$ is a common face, edge, or vertex of $K_{1}$ and $K_{2}$;

4. diam $K \leq h$ for all $K \in \tau_{h}$.

Our family of conforming finite element spaces, $\mathcal{A}_{h}$, defined for mesh diameters in the range $0<h<h_{0}$, satisfies

$$
\mathcal{A}_{h} \subset W^{1, \infty}\left(\Omega ; \mathbb{R}^{3}\right) \quad \text { for } 0<h<h_{0} .
$$

We assume that there exists an interpolation operator $\mathcal{I}_{h}: W^{1, \infty}\left(\Omega ; \mathbb{R}^{3}\right) \rightarrow \mathcal{A}_{h}$ such that

$$
\operatorname{ess}_{\sup } \operatorname{se}_{x \in \Omega}\left\|\nabla \mathcal{I}_{h} y(x)\right\| \leq C \operatorname{ess} \sup _{x \in \Omega}\|\nabla y(x)\|
$$

for all $y \in W^{1, \infty}\left(\Omega ; \mathbb{R}^{3}\right)$, where the constant $C$ in (41) and below will always denote a generic positive constant independent of $h$. We also assume for $y \in W^{1, \infty}\left(\Omega ; \mathbb{R}^{3}\right)$ that

$$
\left.\mathcal{I}_{h} y(x)\right|_{K}=\left.y(x)\right|_{K} \text { for any } K \in \tau_{h} \text { such that }\left.y(x)\right|_{K} \in\left\{P^{1}(K)\right\}^{3},
$$

where $\left\{P^{1}(K)\right\}^{3} \equiv P^{1}(K) \times P^{1}(K) \times P^{1}(K)$ and $P^{1}(K)$ denotes the space of linear polynomials defined on $K$.

We denote the finite element space of admissible functions satisfying the boundary condition

$$
y_{h}(x)=F_{\lambda} x \quad \forall x \in \partial \Omega
$$

by

$$
\mathcal{A}_{h, F_{\lambda}}=\mathcal{A}_{h} \cap W_{F_{\lambda}}^{1, \infty}\left(\Omega ; \mathbb{R}^{3}\right)=\left\{y_{h} \in \mathcal{A}_{h}: y_{h}(x)=F_{\lambda} x \text { for } x \in \partial \Omega\right\},
$$

and we further assume that the interpolation operator $\mathcal{I}_{h}$ satisfies the property that

$$
\mathcal{I}_{h} y \in \mathcal{A}_{h, F_{\lambda}} \quad \text { if } \quad y \in W_{F_{\lambda}}^{1, \infty}\left(\Omega ; \mathbb{R}^{3}\right) .
$$


The most widely used conforming finite element methods based on continuous, piecewise polynomial spaces have interpolation operators $\mathcal{I}_{h}$ satisfying (41) (for quasiregular meshes), (42), and (43) (see [9, 28]). In particular, (41)-(43) are valid for trilinear elements defined on rectangular parallelepipeds as well as for linear elements defined on tetrahedra.

The following theorem gives the existence of finite element energy-minimizers as well as the error estimate of the corresponding minimum energy.

THEOREM 7. There exist a positive constant $C$ and $y_{h} \in \mathcal{A}_{h, F_{\lambda}}$ such that

$$
\mathcal{E}\left(y_{h}\right)=\min _{z_{h} \in \mathcal{A}_{h, F_{\lambda}}} \mathcal{E}\left(z_{h}\right) \leq C h^{1 / 2}
$$

Proof. We have by the quadratic growth rate of the energy density (6) that

$$
\begin{aligned}
& \|\nabla y\|_{L^{2}\left(\Omega ; \mathbb{R}^{3 \times 3}\right)} \leq\|\nabla y-\pi(\nabla y)\|_{L^{2}\left(\Omega ; \mathbb{R}^{3 \times 3}\right)}+\|\pi(\nabla y)\|_{L^{2}\left(\Omega ; \mathbb{R}^{3 \times 3}\right)} \\
& \leq \kappa^{-1 / 2} \mathcal{E}(y)^{1 / 2}+\sqrt{2 \eta_{1}^{2}+\eta_{2}^{2}}(\operatorname{meas} \Omega)^{1 / 2} \quad \forall y \in W_{F_{\lambda}}^{1, \infty}\left(\Omega ; \mathbb{R}^{3}\right),
\end{aligned}
$$

and we have by the Poincaré inequality $[1,31]$, since $y(x)=F_{\lambda} x$ for all $x \in \partial \Omega$ for $y \in W_{F_{\lambda}}^{1, \infty}\left(\Omega ; \mathbb{R}^{3}\right)$, that

$$
\|y\|_{W^{1,2}\left(\Omega ; \mathbb{R}^{3}\right)} \leq C\|\nabla y\|_{L^{2}\left(\Omega ; \mathbb{R}^{3 \times 3}\right)}+C \quad \forall y \in W_{F_{\lambda}}^{1, \infty}\left(\Omega ; \mathbb{R}^{3}\right) .
$$

We thus obtain from (45) and (46) the growth property that

$$
\|y\|_{W^{1,2}\left(\Omega ; \mathbb{R}^{3}\right)} \leq C \mathcal{E}(y)^{1 / 2}+C \quad \forall y \in W_{F_{\lambda}}^{1, \infty}\left(\Omega ; \mathbb{R}^{3}\right) .
$$

The existence of an finite element energy minimizer $y_{h} \in \mathcal{A}_{h, F_{\lambda}}$ now follows by compactness from the continuity of $\mathcal{E}$ restricted to the finite-dimensional affine space $\mathcal{A}_{h, F_{\lambda}}$ and the growth property of the energy (47).

To finish the proof, we refer to $[8,21]$ for the construction of a finite element deformation $z_{h} \in \mathcal{A}_{h, F_{\lambda}}$ such that

$$
\mathcal{E}\left(z_{h}\right) \leq C h^{\frac{1}{2}}, \quad 0<h<h_{0}
$$

The number of local minima of the problem

$$
\inf _{v_{h} \in \mathcal{A}_{h, F_{\lambda}}} \mathcal{E}\left(v_{h}\right)
$$

grows arbitrarily large as the mesh size $h \rightarrow 0$ [20]. Many of these local minima are approximations on different length scales to the same optimal microstructure [20]. Thus, it is reasonable to give error estimates for finite element approximations $y_{h} \in \mathcal{A}_{h, F_{\lambda}}$ satisfying the quasi-optimality condition

$$
\mathcal{E}\left(y_{h}\right) \leq \alpha \inf _{z_{h} \in \mathcal{A}_{h, F_{\lambda}}} \mathcal{E}\left(z_{h}\right)
$$

for some constant $\alpha>1$ independent of $h$.

It follows directly from the above theorem and the bounds established in sections 3,4 , and 5 that we can obtain the following error estimates for a quasi-optimal finite element deformation $y_{h} \in \mathcal{A}_{h, F_{\lambda}}$.

COROLlaRY 1. If $w \in \mathbb{R}^{3}$ satisfies $w \cdot n=0$, then there exists a positive constant C such that

$$
\int_{\Omega}\left|\left[\nabla y_{h}(x)-F_{\lambda}\right] w\right|^{2} d x \leq C h^{1 / 4}
$$

for any $y_{h} \in \mathcal{A}_{h, F_{\lambda}}$ which satisfies the quasi-optimality condition (48). 
COROLlaRY 2. There exists a positive constant $C$ such that

$$
\int_{\Omega}\left|y_{h}(x)-F_{\lambda} x\right|^{2} d x \leq C h^{1 / 4}
$$

for any $y_{h} \in \mathcal{A}_{h, F_{\lambda}}$ which satisfies the quasi-optimality condition (48).

Corollary 3. If $\omega \subset \Omega$ is a Lipschitz domain, then there exists a positive constant $C$ such that

$$
\left\|\int_{\omega}\left[\nabla y_{h}(x)-F_{\lambda}\right] d x\right\| \leq C h^{1 / 16}
$$

for any $y_{h} \in \mathcal{A}_{h, F_{\lambda}}$ which satisfies the quasi-optimality condition (48).

COROLlaRY 4 . There exists a positive constant $C$ such that

$$
\int_{\Omega}\left\|\nabla y_{h}(x)-\Pi\left(\nabla y_{h}(x)\right)\right\|^{2} d x \leq C h^{1 / 4}
$$

for any $y_{h} \in \mathcal{A}_{h, F_{\lambda}}$ which satisfies the quasi-optimality condition (48).

COROLlarY 5. If $\omega \subset \Omega$ is a Lipschitz domain and $\rho>0$, then there exists a positive constant $C$ such that

$$
\left|\frac{\operatorname{meas} \omega_{\rho}^{0}\left(y_{h}\right)}{\operatorname{meas} \omega}-(1-\lambda)\right|+\left|\frac{\operatorname{meas} \omega_{\rho}^{1}\left(y_{h}\right)}{\operatorname{meas} \omega}-\lambda\right| \leq C h^{1 / 16}
$$

for any $y_{h} \in \mathcal{A}_{h, F_{\lambda}}$ which satisfies the quasi-optimality condition (48).

COROLlaRY 6 . There exists a positive constant $C$ such that

$$
\left|\int_{\Omega}\left\{f\left(x, \nabla y_{h}(x)\right)-\left[(1-\lambda) f\left(x, F_{0}\right)+\lambda f\left(x, F_{1}\right)\right]\right\} d x\right| \leq C\|f\|_{\mathcal{V}} h^{1 / 8}
$$

for any $f \in \mathcal{V}$ and any $y_{h} \in \mathcal{A}_{h, F_{\lambda}}$ which satisfies the quasi-optimality condition (48).

\section{REFERENCES}

[1] R. Adams, Sobolev Spaces, Academic Press, New York, 1975.

[2] J. Ball And R. James, Fine phase mixtures as minimizers of energy, Arch. Rational Mech. Anal., 100 (1987), pp. 13-52.

[3] J. Ball And R. James, Proposed experimental tests of a theory of fine microstructure and the two-well problem, Philos. Trans. Roy. Soc. Lond. Ser. A, 338 (1992), pp. 389-450.

[4] B. Brighi and M. Chipot, Approximated convex envelope of a function, SIAM J. Numer. Anal., 31 (1994), pp. 128-148.

[5] C. Carstensen and P. Plecháč, Numerical solution of the scalar double-well problem allowing microstructure, Math. Comp., 66 (1997), pp. 997-1026.

[6] M. Снipot, Numerical analysis of oscillations in nonconvex problems, Numer. Math., 59 (1991), pp. 747-767.

[7] M. Chipot And C. Collins, Numerical approximations in variational problems with potential wells, SIAM J. Numer. Anal., 29 (1992), pp. 1002-1019.

[8] M. Chipot, C. Collins, And D. Kinderlehrer, Numerical analysis of oscillations in multiple well problems, Numer. Math., 70 (1995), pp. 259-282.

[9] P. Ciarlet, The Finite Element Method for Elliptic Problems, North-Holland, Amsterdam, 1978.

[10] C. Collins, D. Kinderlehrer, And M. Luskin, Numerical approximation of the solution of a variational problem with a double well potential, SIAM J. Numer. Anal., 28 (1991), pp. 321-332. 
[11] C. Collins AND M. Luskin, The computation of the austenitic-martensitic phase transition, in Partial Differential Equations and Continuum Models of Phase Transitions, M. Rascle, D. Serre, and M. Slemrod, eds., Lecture Notes in Physics 344, Springer-Verlag, New York, 1989, pp. 34-50.

[12] C. Collins And M. Luskin, Optimal order estimates for the finite element approximation of the solution of a nonconvex variational problem, Math. Comp., 57 (1991), pp. 621-637.

[13] J. ERICKSEn, Constitutive theory for some constrained elastic crystals, J. Solids Structures, 22 (1986), pp. 951-964.

[14] D. FRENCH, On the convergence of finite element approximations of a relaxed variational problem, SIAM J. Numer. Anal., 28 (1991), pp. 419-436.

[15] P. Gremaud, Numerical analysis of a nonconvex variational problem related to solid-solid phase transitions, SIAM J. Numer. Anal., 31 (1994), pp. 111-127.

[16] R. James And D. Kinderlehrer, Theory of diffusionless phase transitions, in Partial Differential Equations and Continuum Models of Phase Transitions, M. Rascle, D. Serre, and M. Slemrod, eds., Lecture Notes in Physics 344, Springer-Verlag, New York, 1989, pp. 5184.

[17] P. KLOUČEK AND M. Luskin, The computation of the dynamics of martensitic microstructure, Contin. Mech. Thermodyn., 6 (1994), pp. 209-240.

[18] P. KLOUČEK AND M. Luskin, Computational modeling of the martensitic transformation with surface energy, Math. Comput. Modelling, 20 (1994), pp. 101-121.

[19] R. Kohn, Relaxation of a double-well energy, Contin. Mech. Thermodyn., 3 (1991), pp. 193236.

[20] M. Luskin, On the computation of crystalline microstructure, Acta Numerica, 5 (1996), pp. 191-258.

[21] M. Luskin, Approximation of a laminated microstructure for a rotationally invariant, double well energy density, Numer. Math., 75 (1997), pp. 191-258.

[22] B. Li AND M. Luskin, Nonconforming finite element approximation of crystalline microstructure, Math. Comp., 1998, to appear.

[23] M. Luskin AND L. MA, Analysis of the finite element approximation of microstructure in micromagnetics, SIAM J. Numer. Anal., 29 (1992), pp. 320-331.

[24] L. Ma And N. Walkington, On algorithms for nonconvex optimization, SIAM J. Numer. Anal., 32 (1995), pp. 900-923.

[25] R. A. Nicolaides and N. Walkington, Strong convergence of numerical solutions to degenerate variational problems, Math. Comp., 64 (1995), pp. 117-127.

[26] P. Pedregal, Numerical approximation of parametrized measures, Numer. Funct. Anal. Optim., 16 (1995), pp. 1049-1066.

[27] P. Pedregal, On the numerical analysis of non-convex variational problems, Numer. Math., 74 (1996), pp. 325-336.

[28] A. Quarteroni And A. Valli, Numerical Approximation of Partial Differential Equations, Springer-Verlag, Berlin, 1994.

[29] T. RouBíČEK, A note about relaxation of vectorial variational problems, in Calculus of Variations, Applications and Computations, C. Bandle, ed., Pitman Res. Notes Math. Sci. 326, Longmann, Harlow, UK, 1995, pp. 208-214.

[30] T. RouBíčEK, Numerical approximation of relaxed variational problems, J. Convex Anal., to appear.

[31] J. WlOKA, Partial Differential Equations, Cambridge University Press, Cambridge, UK, 1987. 